\title{
Considerations for the Deep X-ray Lithography with the SU-8 Resist
}

\author{
Laurence Singleton, Maria Kufner and Stephan Megtert ${ }^{a}$ \\ Institut für Mikrotechnik Mainz GmbH, Carl-Zeiss-Str. 18-20, D-55129 Mainz, Germany \\ ${ }^{a}$ Centre Universitaire Paris-Sud, Bâtiment 209 D, F-91405 Orsay-Cedex, France \\ singleton@imm-mainz.de; www.imm-mainz.de
}

\begin{abstract}
It is shown, that deep X-ray exposures of the SU-8 resist can achieve high resolution with substantially reduced exposure times. Irradiation at the synchrotron source of DCI at Lure (Paris) demonstrated a reduced exposure time for a $600 \mu \mathrm{m}$ thick SU-8, where the dose needed to obtain standing structures was $30 \mathrm{~J} / \mathrm{cm}^{3}$. Critical dimension measurements (CD) of the $600 \mu \mathrm{m} \mathrm{SU-8}$ resist structure have been obtained for the entire height of the structure and X-ray doses of $30 \mathrm{~J} / \mathrm{cm}^{3}$ achieved a CD gain per edge of $+0.5 \mu \mathrm{m}$, while doses of $40 \mathrm{~J} / \mathrm{cm}^{3}$ yielded a CD gain per edge of $0.9 \mu \mathrm{m}$. However, the gain in the CD per edge is critically dependent on the solvent content in the resist. Doses of $40 \mathrm{~J} / \mathrm{cm}^{3}$ into a resist with a 2-4\% residual solvent content yielded CD gains per edge of $0.3 \mu \mathrm{m}$. Moreover, the variation in the $C D$ per edge is less than $0.1 \mu \mathrm{m}$ along the entire height of the structure.

Keywords: SU-8, deep X-ray lithography, PMMA, critical dimension, resolution
\end{abstract}

\section{Introduction}

Communication networks will soon be constructured from optical networks as only optical data transfer will be suitable to accomodate the expected increase in data rates.[1,2] Micromanufacturing techniques have been developed and applied in the last years which are of vital importance for the realization of new optical components necessary for such optical networks. These include fiber splices, fiber connectors, fiber switches and integrated optical splitters. Many such components can be manufactured with the LIGA technique, where LIGA is an acronym for lithography, electroforming and replication. Its important feature is the high accuracy of the methods in the sub micrometer range for structure heights of $1 \mathrm{~mm}$ and higher, with an associated structure roughness in the range of $20-30 \mathrm{~nm}$ rms. In addition, the high accuracy of the method over a long lateral distances, i.e. better than $5 \mu \mathrm{m}$ accuracy over $3 \mathrm{~cm}$ lateral distance, means that mould inserts for fiber optical connectors with 12 fibers can be manufactured with the required accuracy by LIGA. [3]

The accuracy of LIGA is largely derived from the lithography step, which is deep X-ray lithography with synchrotron radiation. The use of synchrotron radiation is the key of this success as it delivers an intense and parallel X-ray photon beam suitable for the one to one shadow printing of a patterned mask into a resist. Owing to the short wavelength range used in this process (0.2-0.3 $\mathrm{nm}$ ), effects like mask edges diffraction, resist absorption and mask to resist proximity effects are dramatically reduced in comparison to Soft X-ray or UV Lithography processes. Therefore, the necessity of the lithography with X-rays is not only to achieve very high aspect ratio resist structures, as X-rays are extremely penetrating, but to also transfer mask patterns with very high resolution into the resist.[4,5]

Polymethylmethacrylate (PMMA) is the resist of choice for deep X-ray lithography. It is a positive tone resists and the action of X-rays is chain scission, i.e. the long PMMA chains are broken down to smaller parts by the $\mathrm{X}$-rays and these can be selectively developed away from the remaining resist. Its chief advantage is that it achieves excellent resolution of the mask pattern with quite low sidewall roughness. However, PMMA has a low sensitivity to X-rays. A $500 \mu \mathrm{m}$ resist requires exposure times of hours rather than minutes, even when using high intensity sources such as synchrotrons. The primary reason for its low sensitivity is that it only consists of low atomic weight carbon, oxygen and hydrogen, and these atoms all have a relatively low sensitivity to $\mathrm{X}$-rays. 
Clearly, one would like to obtain a resist requiring shorter exposure times but at the same time achieving high resolution. The SU-8 resist, supplied by Microchem Corporation, has proved suitable for the production of high aspect ratio microstructures with deep UV lithography. Bogdanov has shown that this SU-8 resist, which includes the heavy atom photoinitiator triphenylsulfonium hexafluoroantimonate, requires a bottom dose of between $30 \mathrm{~J} / \mathrm{cm}^{3}$ and $52 \mathrm{~J} / \mathrm{cm}^{3}$ to achieve standing resist microstructures.[6]

The purpose of this publication is to demonstrate the results of exposures on SU-8 (5) and SU-8 (100) at resist heights between $500 \mu \mathrm{m}$ and $600 \mu \mathrm{m}$. In particular, the critical dimensions of the achieved structures are determined, and the realized structure and the intended structure are compared.

\section{Background}

\subsection{Synchrotron Radiation Spectra}

Synchrotron radiation is generated in storage ring devices. It is characterized by its high photon densities and the high collimation of the synchrotron light, which is very important for accurate transfer of pattern from mask to resist in deep X-ray lithography. However, the resist heights are large $(500 \mu \mathrm{m}-1500 \mu \mathrm{m})$ and the small beam divergence, which is always present, has an influence on the final structure.[5,7]

The spectral distribution of the synchrotron radiation depends upon the beam energy and ring radius. The spectrum is continuous and the shape is a function of the characteristic wavelength $\lambda_{c}$, which in Angstroms is given by the equation,

$$
\lambda_{c}=5.59 \frac{R}{E^{3}}
$$

where $E(\mathrm{GeV})$ is the energy of the synchrotron and $R$ (meters) is the bending radius. The actual spectral distribution is determined by the function

$$
P(\lambda, t)=\frac{3^{5 / 2}}{16 \pi^{2}} \frac{e^{2} c}{R^{3}}\left(\frac{E}{m c^{2}}\right)^{7}\left(\frac{\lambda_{c}}{\lambda}\right)^{3} \int_{\lambda_{c} / \lambda}^{\infty} K_{5 / 3}(\eta) d \eta
$$

where $K_{5 / 3}$ is a modified Bessel function, and $e$, $m$ and $c$ have the usual values. [8]

The spectra of DCI with windows and filters, determined from these equations, are shown in Fig. 1. These have been calculated from the synchrotron source parameters in table 1 . As seen

\begin{tabular}{|c|c|}
\hline $\mathrm{E}(\mathrm{GeV})$ & 1.85 \\
\hline $\mathrm{B}$ (Tesla) & 1.6 \\
\hline$\lambda_{\mathrm{c}}$ (Angstroms) & 3.37 \\
\hline $\mathrm{I}(\mathrm{mA})$ & 300 \\
\hline
\end{tabular}

Table 1. Synchrotron Source Parameters for DCI.

\begin{tabular}{|c|c|}
\hline Length & $22.5 \mathrm{~m}$ \\
\hline Window & Beryllium \\
\hline Window Thickness & $610 \mu \mathrm{m}$ \\
\hline Other Filters & $\mathrm{Si}_{3} \mathrm{~N}_{4}(2 \mu \mathrm{m})$, \\
& $\mathrm{Kapton}(12 \mu \mathrm{m})$ \\
\hline
\end{tabular}

Table 2 : D45 Beamline Parameters at DCI.

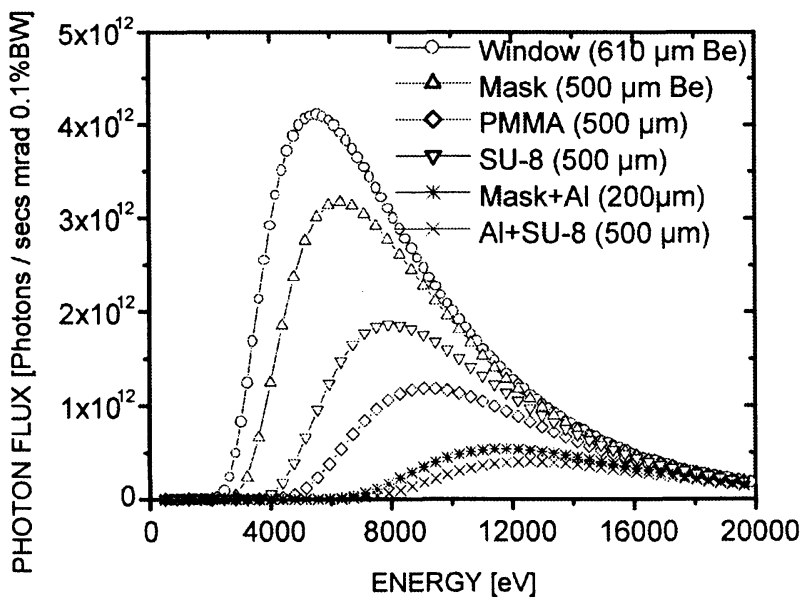

Fig. 1. Influence of beamline filters on the DCI Spectra.

the inclusion of Aluminum filters alters the spectrum drastically.

\subsection{SU-8}

SU-8 is a multifunctional glycidyl ether derivative of a bisphenol-A novolac, containing eight epoxy functionalities on a novolac chain backbone. The photochemical cleavage of an epoxy ring by the photoacid generator during the post exposure bake is subsequently amplified by intramolecular crosslinking to adjacent epoxy rings in the same SU-8 molecule, or by intermolecular crosslinking to the epoxy groups of adjacent SU-8 molecules. This naturally leads to a very extensive chemical response of the initial photochemical step which will lead to a very high crosslinking density of the resist molecules with each other in the post exposure bake. However, it has been previously 


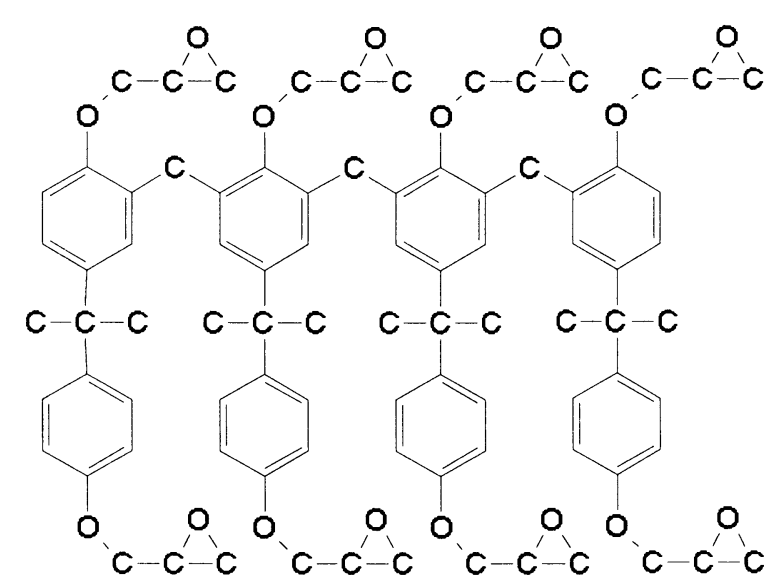

Fig. 2. The SU-8 molecule.

determined by Kupka that the SU- 8 resin seen in fig. 2 (i.e. resist without photoinitiator, supplied by Shell Chemicals) is quite insensitive to X-rays. Here the required bottom dose is $7 \mathrm{~kJ} / \mathrm{cm}^{3}$.[9] The SU-8 resist on the other hand achieves the required resist structures with a bottom dose of some $30 \mathrm{~J} / \mathrm{cm}^{3}$ at DCI. This small dose, compared to $4000 \mathrm{~J} / \mathrm{cm}^{3}$ required by PMMA, is due to the high $\mathrm{X}$-ray sensitivity of antimony atom ( $\mathrm{Sb}$ ) in the photoinitiator, which dramatically increases the transfer of X-ray dose into the resist.

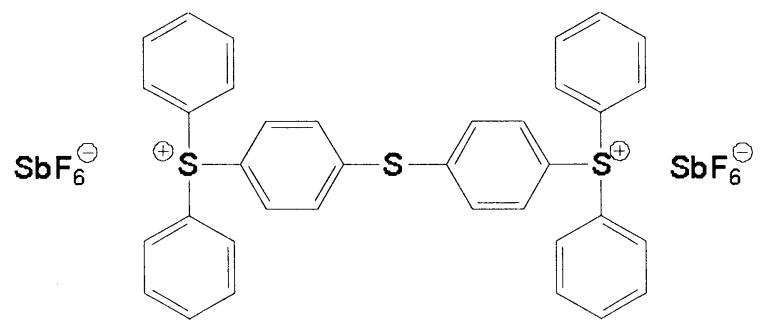

Fig 3. Triarylsulfonium hexafluoroantimonate photoinitiator in the SU-8 resist.

The antimony atom is a heavy atom, which generally have higher sensitivity to X-rays than the lighter atoms, such as carbon oxygen and hydrogen which are found in PMMA. However, as fig. 4 shows, the attenuation coefficient of antimony, which is a measure of the rate of absorption by the element of X-rays, in the wavelength range of interest, is very high. It is significantly higher than that of carbon, the principal element of PMMA, and surprisingly, it is even somewhat greater than that of gold, which is the absorber material

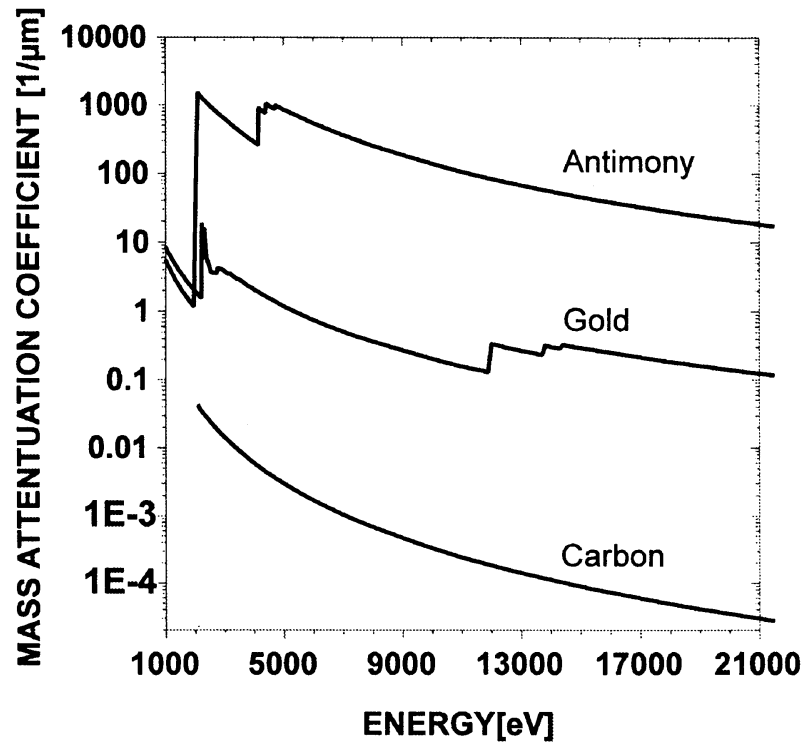

Fig 4. Mass attentuation coefficients of carbon, gold and antimony (Source : NIST).

commonly used in X-ray masks for deep X-ray lithography.

\subsection{Dose Profile in SU-8}

The sensitivity of the antimony to X-rays influences the X-ray dose profile in the resist. The dose profile indicates the rate of transfer of power from the synchrotron radiation to the resist and gives the change in the dose distribution in the resist along its height (fig. 5). So intuitively one expects that the dose absorbed at the top of the resist is greater than that absorbed at the bottom of the resist. This is the case because the lower energy $\mathrm{X}$-rays, have a shorter penetration depth, and they

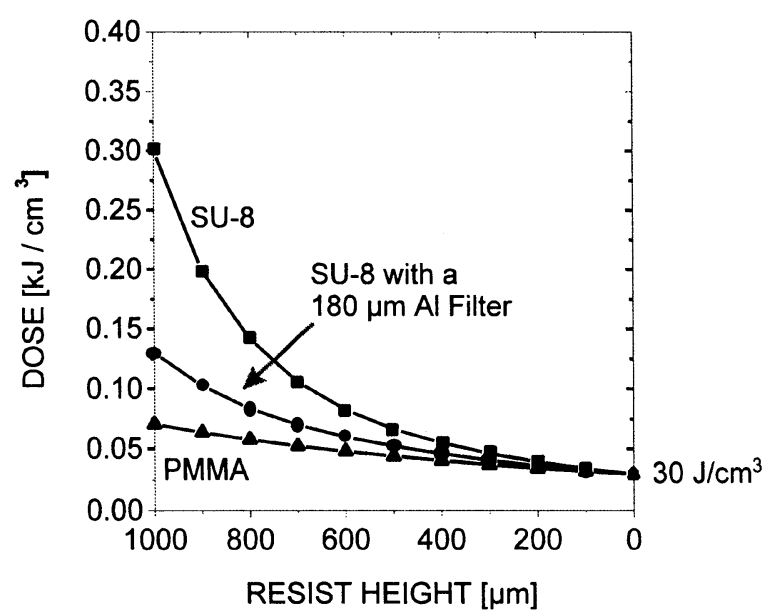

Fig 5: Deposited dose profile in PMMA and SU-8 at DCI 
are absorbed more easily at the resist surface. For low sensitive resists like PMMA, the dose at the top of the resist is only two or three times greater than the dose at the bottom of the resist. For a highly sensitive resist like SU-8, the transfer of synchrotron radiation power at the top of the resist is some ten times greater at the top of the resist in comparison to the bottom of the resist, for a $1 \mathrm{~mm}$ resist. With filters, such as aluminium, this reduces to a ratio of about five. The Aluminum filter only allows high energy, highly penetrating X-rays though, and the profile becomes consequently more even.

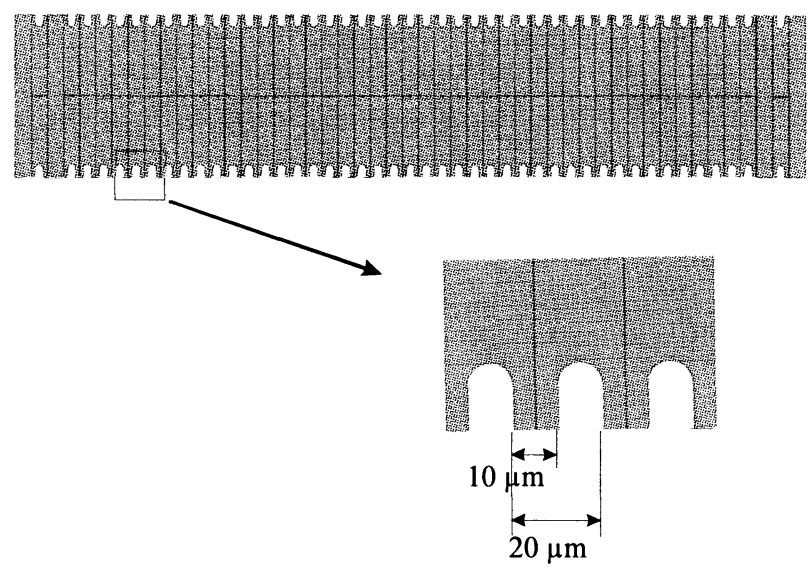

Fig 6. Critical dimension measurement structures

\subsection{Critical Dimension Measurements}

Fig. 6 shows the critical dimension measurement structures. The design represents the profile of the measured SU-8 resist structure. The structure width is $10 \mu \mathrm{m}$, and the pitch is $20 \mu \mathrm{m}$. Measurements are made of the side profile of the SU-8 resist structure, and to reduce corner rounding errors a working mask from a high resolution intermediate mask was used. The intermediate mask lithography step was performed with $100 \mathrm{keV}$ electrons. These absorber structures are set parallel and perpendicular to the scanning direction of the mask, to determine the effect of the horizontal and vertical divergence.

After resist processing, these resist structures were examined using scanning electron microscopy. To overcome the intrinsic measurement errors, the pitch between structures calibrates the measurements, as it is not influenced by shrinking or swelling of the resist.

\begin{tabular}{|c|c|c|c|}
\hline $\begin{array}{c}\text { Bottom } \\
\text { Dose } \\
\left(\mathrm{J} / \mathrm{cm}^{3}\right)\end{array}$ & $\begin{array}{c}\text { Top Dose } \\
\left(\mathrm{J} / \mathrm{cm}^{3}\right)\end{array}$ & Filters & $\begin{array}{c}\text { Resist } \\
\text { Type }\end{array}$ \\
\hline 30 & 180 & $\begin{array}{c}12.5 \mu \mathrm{m} \\
\text { Kapton }\end{array}$ & $\begin{array}{c}\text { SU-8 } \\
(100)\end{array}$ \\
\hline 30 & 52 & $\begin{array}{c}12.5 \mu \mathrm{m} \\
\text { Kapton } \\
180 \mu \mathrm{m} \\
\text { Aluminum }\end{array}$ & $\begin{array}{c}\text { SU-8 } \\
(100)\end{array}$ \\
\hline 40 & 227 & $\begin{array}{c}125 \mu \mathrm{m} \\
\text { Kapton }\end{array}$ & SU-8 (5) \\
\hline 40 & 70 & $\begin{array}{c}12.5 \mu \mathrm{m} \\
\text { Kapton } \\
180 \mu \mathrm{m} \\
\text { Aluminum }\end{array}$ & $\begin{array}{c}\text { SU-8 } \\
(100)\end{array}$ \\
\hline
\end{tabular}

Table 3. SU-8 Irradiation Parameters for DCI.

\begin{tabular}{|c|c|}
\hline Requirement & Parameter \\
\hline Heat source & Hot Plate \\
\hline Intermediate PEB & $50^{\circ} \mathrm{C}, 5$ mins \\
\hline Final PEB & $90^{\circ} \mathrm{C}, 15$ mins \\
\hline
\end{tabular}

Table 4. Post exposure bake (PEB) parameters.

\begin{tabular}{|c|c|}
\hline Developer & Gammabutyrolactone, \\
\hline First Stage & $\begin{array}{c}30 \text { mins } \\
\text { Gentle agitation }\end{array}$ \\
\hline Second Stage & $\begin{array}{c}10 \text { mins } \\
\text { Gentle agitation }\end{array}$ \\
\hline
\end{tabular}

Table 5. SU-8 development parameters.

Critical dimension (CD) measurements of the structure profile were made for the entire height of the resist. The width of the $10 \mu \mathrm{m}$ column was measured at regular points along the height of the resist. Typical resolution error of the measurement was $0.05 \mu \mathrm{m}$. These measured widths were compared to the mask structure width to yield the critical dimension per structure edge. This simply is the change in the structure relative to the original mask structure.

\section{Experimental}

Tables 3, 4 and 5 show the resist process parameters for irradiation, post exposure bake and development. Samples were prepared in two ways. One followed the method proposed by Bogdanov.[6] The pre-treatment of the wafers 

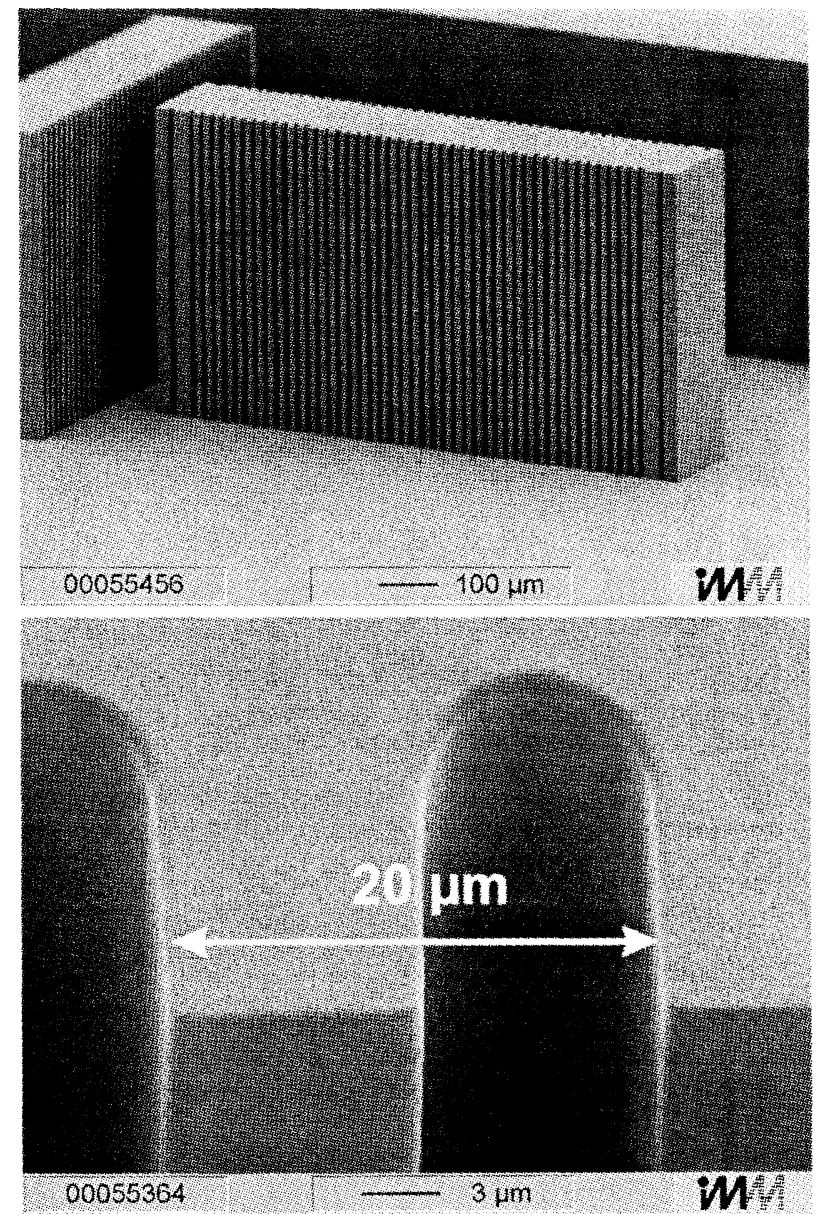

Fig 7. $600 \mu \mathrm{m}$ high SU-8 resist structures with $10 \mu \mathrm{m}$ widths exposed at DCI. Exposure time, 1 minute.

consisted in a dehydration bake for 45 minutes at $250-275^{\circ} \mathrm{C}$ in a vacuum oven. The resist was applied by casting $6.5 \mathrm{mls}$ of the SU-8 (5) resist onto the wafer on the hotplate at a temperature of $90^{\circ} \mathrm{C}$ using a syringe. The probe was kept on the hotplate at $90^{\circ} \mathrm{C}$ for 13 to 14 hours in order to entirely remove the solvent GBL (Gammabutyrolactone). For this cast resist, the measured thickness was between $505 \mu \mathrm{m}$ and $510 \mu \mathrm{m}$.

Alternatively, after dehydration at $250^{\circ} \mathrm{C}$ in a vacuum oven, one can spincoat $6.5 \mathrm{mls}$ of SU-8 (100) resist on a 4 inch Silicon wafer in a low turbulence, solvent saturated environment (Karl Suss CT62) to achieve a $600 \mu \mathrm{m}$ resist in a single spin. Solvent saturation and low turbulence is simply achieved by enclosing the spinning resist in a closed cover environment, and this helps to achieve a more even distribution of the resist over the wafer surface. This is particularly important

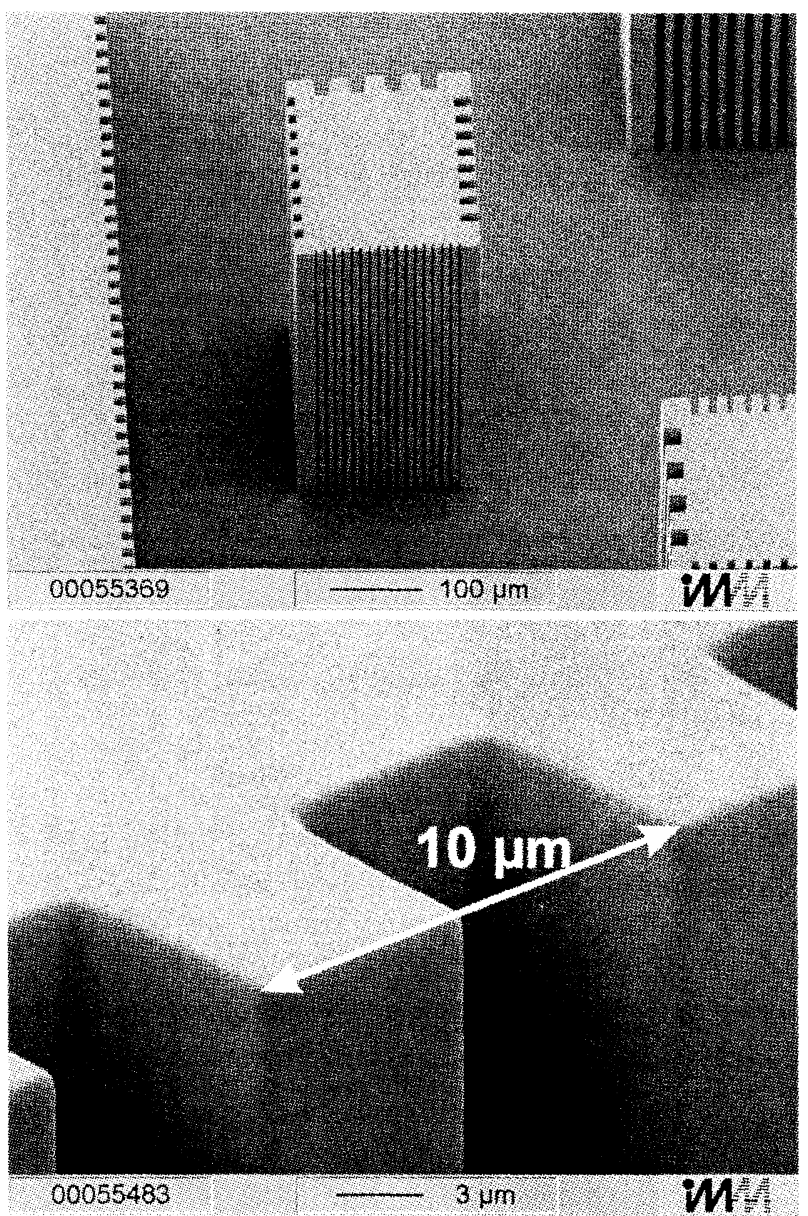

Fig $8600 \mu \mathrm{m}$ high SU-8 resist structures with $5 \mu \mathrm{m}$ widths exposed at DCI. Exposure time, 1 minute.

for viscous resists such as the SU-8 (100). Here, the probe was kept on the hotplate at $90^{\circ} \mathrm{C}$ for 6 to 7 hours, in order to remove the solvent. The resist height was between $580 \mu \mathrm{m}$ and $600 \mu \mathrm{m}$.

Exposures took place at the Jenoptik scanner attached to the D45 beamline at DCI. A bottom dose of $30 \mathrm{~J} / \mathrm{cm}^{3}$ was sufficient to achieve standing resist structures (fig. 7 and fig. 8). Post exposure bake followed immediately after irradiation and after cooling, the resist was developed in GBL, in a two stage immersion development. The probe was then washed with isoproponal, and left to dry in air.

\section{Results and Discussion}

\subsection{Results}

Exposures of a $600 \mu \mathrm{m}$ SU-8 resist at DCI require a bottom dose of only $30 \mathrm{~J} / \mathrm{cm}^{3}$ to achieve efficient pattern transfer throughout the depth of the resist. In comparison, PMMA demands a 


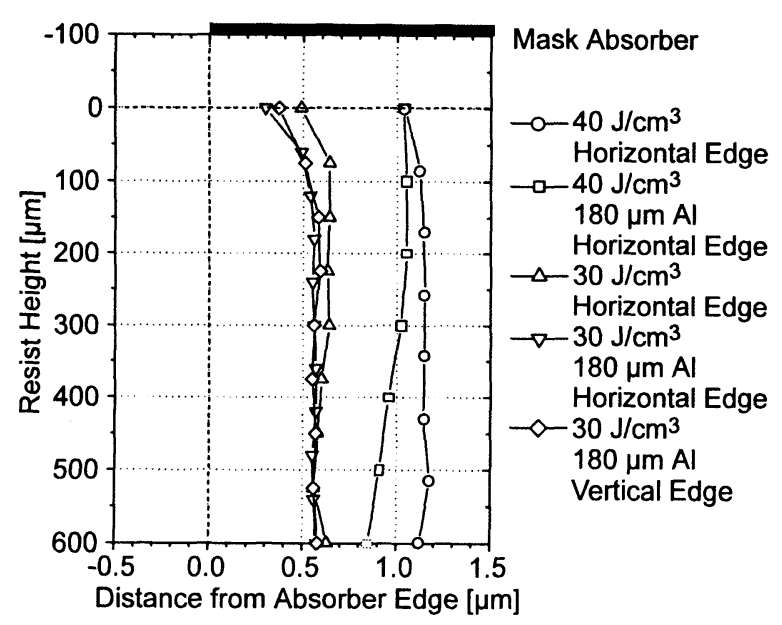

Fig. 9. Critical dimension measurements of $600 \mu \mathrm{m}$ SU-8 resist.

bottom dose in excess of $4 \mathrm{~kJ} / \mathrm{cm}^{3}$ for a DCI exposure to achieve an equivalent pattern transfer.

Critical dimension measurements were made on the exposed structures. Fig. 9 shows the $C D$ gain per edge for $600 \mu \mathrm{m}$ high resist structures exposed with a bottom dose of $30 \mathrm{~J} / \mathrm{cm}^{3}$. One set of exposures were performed without an Aluminum filter and the others with a $180 \mu \mathrm{m}$ Aluminum filter in the beamline. The gain in the CD per edge is on average $+0.55 \mu \mathrm{m}$. No dependence on the dose profile nor the direction of the absorber edge, whether it be horizontal or vertical, was observed in the $\mathrm{CD}$ per edge values.

Fig. 9 also shows the $C D$ gain per edge for structures exposed with a $40 \mathrm{~J} / \mathrm{cm}^{3}$ bottom dose. Here, the $C D$ gain per edge has increased to an average of $+0.9 \mu \mathrm{m}$. As before for the $30 \mathrm{~J} / \mathrm{cm}^{3}$ exposures, the inclusion of Aluminum filters does not significantly influence the $C D$. gain per structure edge.

Fig. 10 shows similar $C D$ changes per structure edge, for exposures with bottom doses of $40 \mathrm{~J} / \mathrm{cm}^{3}$. Both of these exposures were performed in $510 \mu \mathrm{m}$ resist cast from SU-8 (5), with a softbake of $90^{\circ} \mathrm{C}$ in excess of 13 hours. The CD per edge for the $40 \mathrm{~J} / \mathrm{cm}^{3}$ exposure is $+0.3 \mu \mathrm{m}$. As before, these exposures show no dependence on the absorber edge direction.

\subsection{Discussion}

From fig 9 it is to be seen clearly that the use of Aluminum filters nor the change in divergence results in a significant influence on the critical dimension gain per edge. The influence must come from the dose, as the two separate

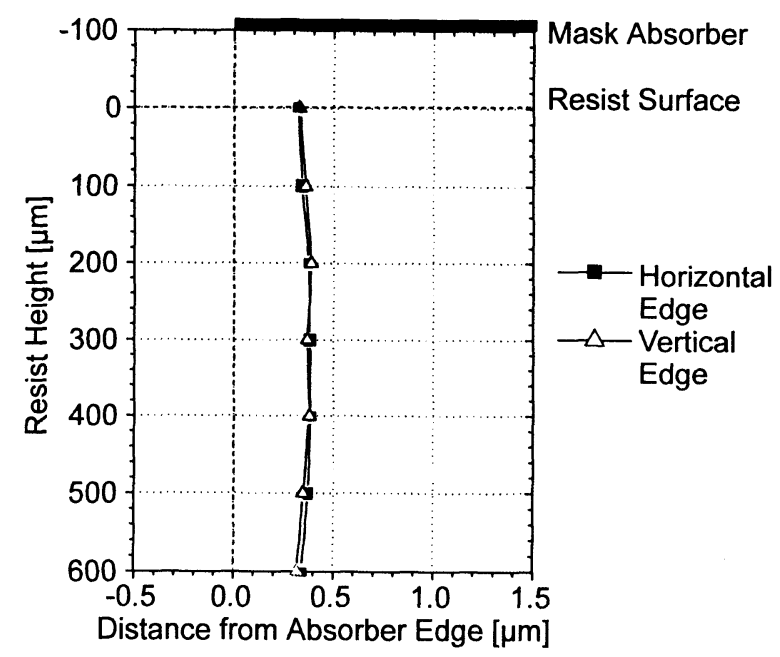

Fig. 10. Critical dimension measurements of $600 \mu \mathrm{m}$ SU-8 resist.

measurements differ significantly for the bottom dose of the irradiated resist. Therefore, the biggest contribution to the influence of the critical dimension comes from the irradiated dose in the resist.

However, fig. 10 shows that the dose alone is not the arbitrating factor. The probe in this case received a 13 hour softbake, and the previous samples obtained a 7 hour softbake.

Fig.11 shows the amount of solvent remaining in the resist after softbake. The weight of the resist and substrate is measured over a period of hours, to obtain the total resist and solvent weight. The final

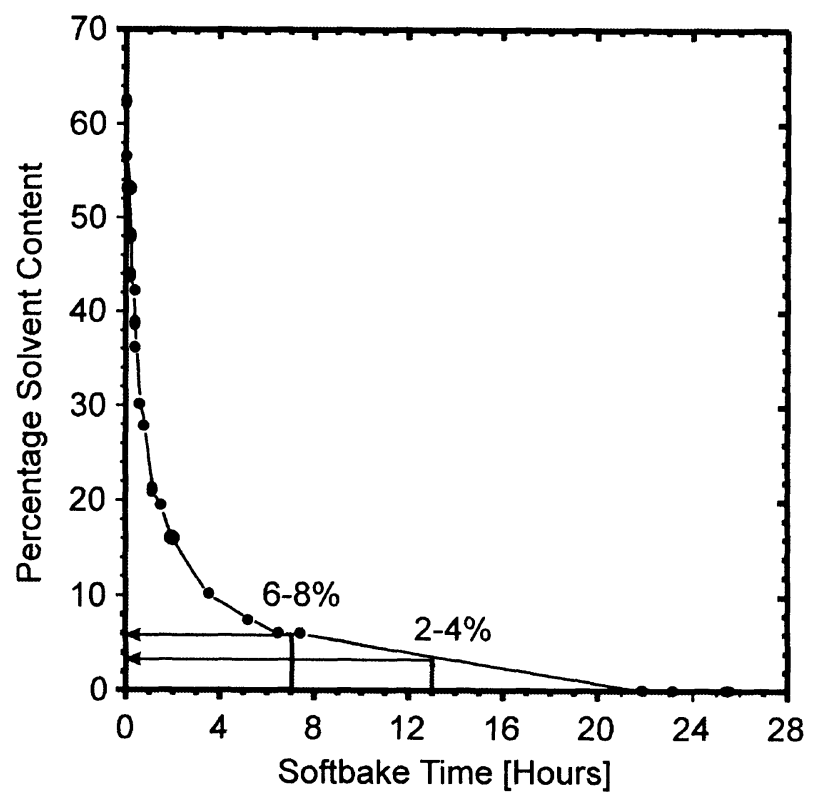

Fig. 11. Percentage solvent remaining in resist versus softbake time (source: S. Peredkov, Lund) 
weight of the resist is taken as the constant weight after 25 hours softbake time, and from this weight, the percentage of solvent still remaining in the resist is determined. As can be seen, the solvent content remaining after a 7 hour softbake is approximately $8 \%$, while after 12 or thirteen hours softbake, the solvent content is between $2-4 \%$ of the total weight.

The principal crosslinking reaction of SU-8 only proceeds in the post exposure bake. This requires a movement of the photo generated acid to achieve crosslinking.[10] Clearly from the data shown here, this movement of acid, which will certainly affect the critical dimension, is influenced by the remaining solvent content. So by reducing the solvent content, one reduces the change in the critical dimension. Therefore, the influence on the final structure dimension cannot be alone a factor of deposited dose, but it is also a factor influenced by the diffusion of the photoacid during the post exposure bake.

\section{Simulation}

The simulation performed here considered only three aspects of possible influences to the critical dimension change. These were divergence, diffraction and secondary electron effects. Diffraction at a semitransparent absorber edge was calculated in the Fresnel approximation. A Gaussian angular distribution of the electrons with a standard deviation of $0.2 \mathrm{mrad}$ and the wavelength dependent natural divergence given by theory was used to calculate the combined dose distribution due to diffraction and divergence. To calculate the image blur caused by the photoelectrons and the Auger electrons a Monte Carlo simulation has been performed. In order to achieve reasonable computing times the Monte Carlo data have been parametrized by fitting the monochromatic results with a Gaussian distribution. The dose deposition due to diffraction, synchrotron radiation divergence and electron energy deposition was calculated by convolution. The contribution of the image blur caused by the electrons is much larger than the diffraction and divergence effects. This result holds for resist layers with a thickness of more than some 10 $\mu \mathrm{m} .[5]$

Fig. 12 shows the the calculated isodosis lines with the inclusion of diffraction, divergence and secondary electron effects. In this case the bottom dose for the irradiation at DCI was $40 \mathrm{~J} / \mathrm{cm}^{3}$. This

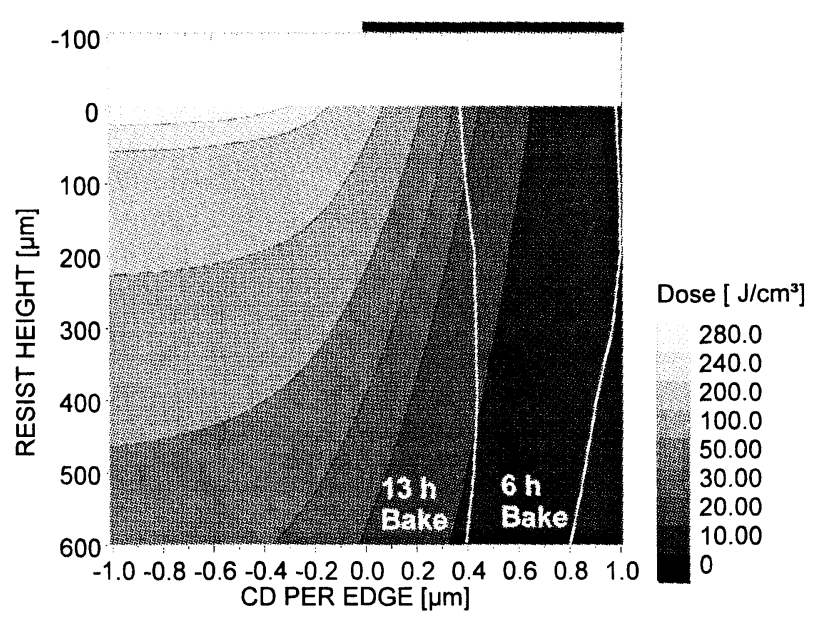

Fig. 12. Simulate dose deposition in SU-8, and the measured accuracy for different softbake times.

model dose not yet include the effects of the post exposure bake nor the effects of development, both which would have in this case an important influence on the final structure. Fig. 12 also includes the measured structural accuracy for structures irradiated at this bottom dose. The two curves show the changes for the resist having different softbake times. Although it is to be expected, that the absence of the post exposure bake model leads to inaccuracy in the comparison, the isodosis lines do not concur with the observed structure curve. That is to say that the change in the structure curve is less than $0.1 \mu \mathrm{m}$ from the top to the bottom of the structure, but that of the calculated isodosis lines have approximately an $0.2-0.3 \mu \mathrm{m}$ change from the top to the bottom of the resist. Since it would be expected that the PEB model would reflect the a consistent change relative to the isodosis lines, the difference between the calculated and measured results is surprising. In that case, a development model may be necessary, but also some resist compression at the crosslinking stage may also be important in the calculation of the final structures.

\section{Conclusions}

The exposures of SU-8 with synchrotron radiation at DCI (Paris) demonstrates the capability to pattern SU-8 with only very small doses. In this case a $30 \mathrm{~J} / \mathrm{cm}^{3}$ bottom dose is sufficient.

Critical dimension measurements have been performed and a dependence of the critical dimension per structure edge on the deposited dose is observed. A $30 \mathrm{~J} / \mathrm{cm}^{3}$ dose gives an average CD 
change of $+0.55 \mu \mathrm{m}$, while a $40 \mathrm{~J} / \mathrm{cm}^{3}$ dose gives a change of $+0.9 \mu \mathrm{m}$. However, by reducing resist solvent content (longer softbake time) reduced critical dimension changes are seen. In the case here, a softbake time of 13 hours gives an $0.3 \mu \mathrm{m}$ change per edge.

Simulation of the exposure without simulation of the PEB and the development has been used to correlate observed structures. However, the isodosis lines do not concur very well with the observed exposure, so the PEB and development models are important.

\section{Acknowledgements}

This work has been partially supported by a fund from the European Commission, in the project "Microfabrication with Synchrotron Radiation", MICROSYNC, Contract Number ERBFMRXCT97-0140.

\section{References}

1. H.D. Bauer, W. Ehrfeld, J. Hossfeld, T. Paatzsch, NATO -Mediterranean Dialogue Advanced Research Workshop on Unconventional Optical Elements for Information Storage, Processing and Communications, Kiryat Anavim, Israel,(1998) 64.

2. T. Paatzsch, H.D. Bauer, M. Popp, S. Stadler,
I. Smaglinski and W. Ehrfeld, Proceedings of WFOPC'98: Workshop on Fibre Optic Passive Components, (1998) 65.

3. K. Dunkel, H.D. Bauer, W. Ehrfeld, J. Hossfeld, L. Weber, G. Hoercher, G. Mueller, J. Micromech. Microeng. 8 (1998) 301.

4. G. Feiertag VDI Fortschrittberichte, Series 9, Number 242, VDI Verlag (In German).

5. G. Feiertag, W. Ehrfeld, H. Lehr, M. Schmidt, A. Schmidt, Journal of Micromechanics and Microengineering, 7 (1997) 323.

6. A.L. Bogdanov and S.S. Peredkov $25^{\text {th }}$ International Conference on Micro- and NanoEngineering, Rome, Italy, September 21-23 (1999) 493.

7. A. Schmidt, W. Ehrfeld, H. Lehr, Microelectronic Engineering, 30, 1 (1996) 235.

8. H. Winick, Properties of Synchtrotron Radiation, in Synchrotron Radiation Research, Editors, H. Winick, S. Donaich, Plenum Press, New York, 1980.

9. R.K. Kupka, F. Bouamrane, M. Roulliay, and S. Megtert, Proceedings of SPIE 3680 Part1, 508-517, Bellingham, Washington, USA.

10. W. Hinsberg, F. Houle, M. Sanchez, M. Morrisson, G. Wallraff, C. Larson, J. Hoffnagle, P. Brock, and G. Breyta, Proc. SPIE, 3999 (2000) 148. 